\title{
Des espaces frontières d'expérimentation entre pastoralisme et protection de la nature
}

\author{
Céline Granjou, Isabelle Mauz \\ Sociologues, CEMAGREF, UR DTGR, 38402 Saint-Martin-d'Hères cedex, France
}

L'importante littérature sur la gestion négociée entre l'activité agricole et la protection de la nature s'enrichit ici d'une nouvelle analyse dont l'originalité tient à l'usage de la notion d'espace frontière. Elle met l'accent sur l'enjeu que constitue la mise en place de ces espaces d'interface entre plusieurs mondes, laquelle nécessite l'engagement d'acteurs intermédiaires qui prennent le risque d'une expérimentation aussi bien technique que sociale. Le pari d'une telle publication disciplinaire dans NSS tient au fait qu'elle doit être une invitation à la réflexion pour les écologues et les zootechniciens.

La Rédaction

\section{Mots-clés :}

pastoralisme ; parcs naturels ; entrepreneurs de conciliation ; expérimentation sociotechnique ; espaces frontières

\begin{abstract}
Résumé - Cet article entend contribuer à la compréhension sociologique des conditions - et des difficultés - du rapprochement entre le monde agricole et celui de la protection de la nature. À partir d'une enquête sociologique menée dans trois parcs alpins (Bauges, Mercantour, Vanoise), il montre notamment comment des acteurs «entrepreneurs" de conciliation ont constitué des «espaces frontières » où ces deux univers se rencontrent et où se construisent des connaissances et des pratiques de gestion concertée des alpages. Nous analysons les motivations, les trajectoires et les modes d'action de ces acteurs entrepreneurs, ainsi que leurs effets. Nous soulignons la précarité particulière des espaces frontières et caractérisons certaines des difficultés qu'ils rencontrent. Cet article montre ainsi comment l'expérimentation technique (production de connaissances et de pratiques) s'ancre dans une expérimentation sociale (déploiement de trajectoires et d'identités professionnelles).
\end{abstract}

\section{Keywords:}

pastoralism; natural parks; conciliation entrepreneurs, sociotechnical experimentation; boundary areas

\begin{abstract}
Experimenting boundary areas between pastoralism and nature conservation. This article aims to show how an understanding between the worlds of agriculture and nature conservation may be established in some French alpine national or regional parks. Drawing on a sociological survey in three alpine parks (Bauges, Mercantour, Vanoise), we show how local "conciliation entrepreneurs" have created boundary areas between pastoralism and nature conservation where stakeholders can meet and develop new knowledge and practices to manage mountain pastures. We examine the motivations of these entrepreneurs, the way they proceed, their trajectories, and the effects of their action. We underline the precarious nature of these boundary areas and highlight some of the problems they encounter. Our study stresses that producing new knowledge and practices also means producing new professional trajectories and social identities for the local stakeholders.
\end{abstract}

Des mesures agrienvironnementales (Alphandéry et Billaud, 1996) aux politiques de gestion de la biodiversité (Larrère et al., 2009; Blandin, 2009), en passant par Natura 2000 (Pinton, 2006), les initiatives institutionnelles ne manquent pas qui visent à rapprocher les objectifs et les pratiques du monde de la production agricole et de celui de la protection de la nature. Notre objectif est ici de contribuer à la compréhension sociologique des conditions - et des difficultés - d'un tel rapprochement. En effet, davantage qu'à l'imposition systématique d'un dispositif ou d'une norme générale, les dynamiques de conciliation entre monde agricole et monde de la protection de la nature nous paraissent tenir bien souvent à des formes locales d'expérimentation

Auteur correspondant : C. Granjou, celine.granjou@irstea.fr 
sociotechnique (Lascoumes et al., 1997). Plutôt que de prétendre à l'avènement d'un "paradigme conciliateur " généralisé, cet article entend caractériser finement les conditions locales, en partie contingentes, de rapprochement et de coconstruction entre les deux mondes à partir de l'examen empirique des configurations d'action collective prévalant dans différents parcs naturels ${ }^{1}$.

Nous entendons tout particulièrement éclairer le rôleclé joué dans ces configurations par des acteurs " entrepreneurs » de conciliation. Agissant comme des « entrepreneurs de morale» (Becker, 1985), ils défendent la nécessité de trouver une voie intermédiaire entre production agricole et protection de la nature et tentent $\mathrm{d}^{\prime} \mathrm{y}$ enrôler d'autres acteurs, agriculteurs, collègues ou chercheurs travaillant sur les liens entre conservation de la biodiversité et pratiques agricoles ${ }^{2}$. Pour comprendre les conditions et les effets de ces entreprises de conciliation, nous proposons de recourir à la notion $\mathrm{d}^{\prime}$ « espace frontière » («boundary area ») empruntée à la géographie culturelle (Kohler, 2002). Comme les notions issues des Science Studies («boundary work» : Gieryn, 1983 ; «boundary object »: Star et Griesemer, 1989 ; «trading zone» : Galison, 1996 ; «boundary organization » : Guston, 2001), celle-ci théorise le fait que la frontière ne permet pas simplement à deux mondes de se démarquer, mais aussi de se côtoyer et de se rencontrer. Elle vise à caractériser des formes d'échange et de coordination locales qui se dégagent sur un fond où deux univers de valeurs et de pratiques distinctes continuent de prévaloir ${ }^{3}$. À la différence des concepts de Science Studies, la notion d'espace frontière vise non seulement à saisir les connaissances et les pratiques hybrides qui se construisent à l'articulation entre deux mondes (en l'occurrence, des connaissances et des pratiques de gestion concertée des alpages), mais aussi à reconstituer les arènes sociales, les trajectoires professionnelles et les identités des acteurs qui y circulent. Enfin, particulièrement plastique, $c^{\prime}$ est une notion qui permet de décrire et de mettre en parallèle des entreprises de conciliation caractérisées par des degrés divers d'extension et de concrétisation ${ }^{4}$.

1 Ce travail a été conduit avec le soutien financier de l'Agence nationale de la recherche dans le cadre du programme " Agriculture et développement durable », projet Pro-DD.

2 On verra que la qualification d'entrepreneur de morale s'accompagne d'un registre d'action parfois très pragmatique.

3 Les espaces frontières sont pour Kohler (2002, p. 16) « non pas des endroits où le mouvement cesse, mais où il est le plus intense et le plus structurant » (notre traduction).

4 Kohler lui-même entendait moins proposer une théorie des espaces frontières qu'utiliser la richesse heuristique de cette métaphore géographique pour étudier les rapports entre la biologie de terrain et la biologie de laboratoire au tournant du XIX ${ }^{\mathrm{e}}$ siècle.
Nous avons mené l'enquête empirique dans trois parcs alpins (un parc naturel régional :les Bauges, et deux parcs nationaux: la Vanoise et le Mercantour), afin de retracer les configurations locales d'action collective et les dynamiques de conciliation entre monde agricole et monde de la protection de la nature. Notre enquête, menée de 2006 à 2008, est fondée sur trente-cinq entretiens semi-directifs avec des agents des parcs, des membres de leurs conseils scientifiques, des techniciens et professionnels agricoles ainsi que des chercheurs, complétés par des observations participantes de réunions et par l'exploitation de documents (documents d'objectifs Natura 2000, chartes de parc, comptes rendus de conseils scientifiques des parcs nationaux).

En nous appuyant également sur des emprunts à l'anthropologie symétrique (Akrich et al., 2006), nous montrerons d'abord qui sont ces acteurs entrepreneurs de conciliation, à partir de quelles motivations, de quelles positions et avec quels effets ils se sont investis dans la constitution d'espaces frontières. Nous montrerons ensuite que ces espaces restent souvent fragiles, précaires, voire éphémères. Nous caractériserons alors les difficultés particulières que posent leur extension et leur pérennisation en identifiant les tensions propres au vécu des acteurs entrepreneurs, ainsi que les difficultés de la production de connaissances sur les relations entre pratiques agricoles et maintien de la biodiversité.

\section{Des acteurs entrepreneurs de conciliation}

\section{Trois espaces protégés}

Nous avons mené l'enquête dans deux parcs nationaux (Mercantour et Vanoise) et un parc naturel régional (Bauges). Nous nous sommes intéressées tout particulièrement à certains événements récents symptomatiques, dans les trois cas, de tentatives de conciliation entre les parcs et la profession agricole.

\section{Les rencontres du Mercantour}

La création du Parc national du Mercantour en 1979 a suscité une vive opposition des populations locales et les relations ont d'emblée été difficiles avec les professionnels de l'élevage. Au début des années 1990, l'arrivée des loups dans le massif - le premier des Alpes françaises à être colonisé - a aiguisé les tensions : les agents de terrain se sont fortement impliqués dans la défense du prédateur. Des conflits violents ont éclaté, allant jusqu'à des agressions d'agents du parc par des éleveurs exaspérés par les dégâts. En 2002, dans un climat un peu apaisé, le parc a recruté son premier chargé de mission pastoralisme, qui 
a effectué un important travail de médiation entre les professionnels du pastoralisme et les agents de terrain (sur l'évolution de la position du Parc national du Mercantour par rapport au pastoralisme, voir Laslaz, 2006). C'est avec l'arrivée, en 2004, d'un nouveau directeur du parc que l'initiative a été lancée d'organiser des journées de rencontres avec des scientifiques, dans le but de construire une vision partagée des effets environnementaux des pratiques d'élevage. L'événement a réuni des chercheurs, des gestionnaires de la nature et des professionnels de l'élevage. Du fait de l'affluence du public, elles ont généralement été considérées comme le signe d'un certain rapprochement entre le parc et les éleveurs.

\section{Le Schéma de gestion des espaces agricoles en Vanoise}

Si les premiers agents de terrain du Parc national de la Vanoise, de par leurs origines locales et souvent paysannes, étaient proches des éleveurs, les relations se sont tendues à la fin des années 1980, notamment après que la création d'un concours national en 1989 eut entraîné le recrutement de gardes issus d'un milieu urbain. Cependant, comme dans le cas du Mercantour, une reconnexion du parc au monde agricole s'est faite à l'occasion du recrutement d'un premier chargé de mission pastoralisme en 2001. Dès le milieu des années 1990, un poste « hybride », payé par le parc mais hébergé à la chambre d'agriculture, avait été créé et avait débouché sur une opération locale agrienvironnementale impliquant certains agriculteurs et le parc. Une démarche concertée de rédaction d'un Schéma de gestion des espaces agricoles (SGEA) a été lancée en 2003 avec la chambre d'agriculture, afin de recenser et hiérarchiser les enjeux agricoles et les enjeux environnementaux sur les différents alpages du parc. Le SGEA s'est fait en parallèle avec la rédaction du document d'objectifs Natura 2000 dont le parc est opérateur, visant également à définir des pratiques qui soient à la fois respectueuses des milieux sensibles et acceptables par les éleveurs en termes de revenu et de pénibilité du travail.

\section{Le pâturage en milieu embroussaillé dans les Bauges}

Sur le massif des Bauges, la Réserve nationale de chasse et de faune sauvage a œuvré avec succès, après les années 1950, à restaurer et à développer les populations de grands ongulés sauvages (chamois) en excluant l'activité de pâturage domestique. La création du Parc naturel régional du Massif des Bauges en 1995 a contribué à redistribuer les cartes. D'une part, les éleveurs souhaitent « reconquérir » les alpages situés dans la réserve, car ils ont besoin de terrains supplémentaires de pâturage du fait, en particulier, du succès de l'appellation d'origine contrôlée (tomme des Bauges). D'autre part, nombre de gestionnaires d'espaces et d'élus locaux voient de plus en plus dans la déprise agricole le vecteur d'une détérioration de la biodiversité et des paysages. Deux dispositifs d'action publique sont mis en place dans les années 1990 afin de soutenir l'élevage au nom de ses effets bénéfiques sur l'ouverture des milieux : le Plan local de gestion de l'espace (PLGE) et Natura 2000, qui prévoient à la fois des contrats agrienvironnementaux et le financement de travaux d'équipement (pistes, points d'eau) pour encourager le maintien ou la reprise de l'activité d'élevage sur certains secteurs. Pour mettre en œuvre ces dispositifs, le parc a recruté un chargé de mission, initialement chargé de mission Natura 2000, puis chargé de mission pastoralisme.

\section{La constitution d'espaces frontières}

Sur les territoires des parcs, le souhait des agriculteurs $\mathrm{d}$ 'intensifier leurs pratiques pour garantir leur revenu ou diminuer la pénibilité du travail est généralement jugé comme une menace pour des milieux fragiles, du point de vue environnemental. Pourtant, dans les trois cas observés, un rapprochement entre les milieux de protection de la nature et la profession agricole s'est récemment esquissé. Ce rapprochement résulte en particulier de l'action des chargés de mission pastoralisme ${ }^{5}$, qui ont mené un travail actif d'intéressement et de recrutement dans une voie de conciliation entre les positions environnementales et agricoles.

En défendant le bien-fondé d'une voie intermédiaire conciliant pastoralisme et protection de la nature au détriment des positionnements radicaux, ces acteurs ont joué un rôle d'entrepreneurs. Disposant d'une familiarité avec le monde agricole liée à une formation en agronomie ou à une expérience professionnelle antérieure, ils étaient aussi profondément convaincus de la pertinence et de la validité d'une ouverture à la coopération entre profession agricole et protection de l'environnement. L'envie de travailler à l'articulation entre la protection de la nature et l'agriculture pour faire se « croiser les regards » et développer une « reconnaissance mutuelle » se retrouve ainsi dès leurs choix d'études et de formation. L'un d'eux témoigne : «[...] entre ces deux extrêmes [l'environnementalisme radical de certains agents du parc et la méfiance des agriculteurs vis-à-vis du parc], je pense qu'on doit pouvoir trouver une voie, et qui pour moi, je suis certain, est une voie de demain. » La dimension créative et expérimentale de leur travail, dans des postes explicitement dédiés au dialogue avec la profession agricole, a constitué - au moins dans un premier temps une expérience positive : plusieurs d'entre eux insistent

\footnotetext{
5 Si, dans les cas étudiés, les initiatives de conciliation ont été impulsées par les acteurs des parcs, elles peuvent tout aussi bien provenir, dans d'autres cas, des acteurs agricoles.
} 
sur la dimension de « test », de « brouillon », de démarche coconstruite, et témoignent de l'intérêt qu'ils trouvent à manipuler des sujets qui demandent une réflexion au cas par cas sur le long terme.

Les acteurs entrepreneurs de conciliation ont moins cherché à produire des normes et des règles systématiques qu'à aboutir à des arrangements pratiques et contextuels. L'observation de réunions dans le cas des Bauges a, par exemple, permis de mesurer l'efficacité d'un principe d'action en familiarité (autour d'un apéritif offert par l'éleveur), revendiqué par le chargé de mission lui-même, qui explique s'efforcer de faciliter le dialogue en situation plutôt que de formaliser des accords généraux et officiels. De même, les organisateurs des rencontres du Mercantour et les promoteurs du SGEA en Vanoise se sont avant tout attachés à désamorcer les possibilités d'opposition frontale plutôt qu'à obtenir le ralliement consensuel à une position qui clôturerait le débat. Le SGEA prévoit un principe d'examen des pratiques souhaitables au cas par cas lors de diagnostic pastoraux, sans s'avancer sur un corpus de règles systématiques qui risquerait de provoquer l'opposition de certains représentants agricoles ou de certains agents $d u$ parc. Les rencontres du Mercantour, pensées au départ comme un colloque scientifique, ont été renommées pour mettre l'accent sur la volonté de construire une « vision partagée » des enjeux du territoire, et convaincre les acteurs agricoles qu'il ne s'agissait pas de « liquider » le pastoralisme. Elles n'avaient donc pas pour vocation de formuler des normes d'action fondées sur un savoir irréfutable, mais bien de constituer un lieu de rencontres et de désamorcer les tensions qui avaient prévalu, en particulier depuis le retour du loup dans les années 1990, entre parc et monde agricole.

Pour légitimer et instruire les possibilités de conciliation entre pastoralisme et maintien de la biodiversité, les chargés demission ont également mobilisé des chercheurs afin de tenter de produire des connaissances sur les liens entre pratiques agricoles et maintien de la biodiversité. Le recours aux chercheurs n'est certes pas nouveau sur les territoires des parcs (Selmi, 2006). Cependant, face à la difficulté de construire des indicateurs généraux d'évaluation des effets des pratiques pastorales sur la biodiversité (Encadré 1), les entrepreneurs d'espaces frontières ont mis en œuvre des dynamiques de production et de partage de connaissances scientifiques sur cette question. C'était ainsi l'un des objectifs des rencontres organisées par le Parc national du Mercantour ; les diagnostics pastoraux prévus par le SGEA et Natura 2000 en Vanoise utilisent les résultats de guides techniques produits par des chercheurs travaillant sur le massif depuis plusieurs années; enfin, le Parc naturel régional du Massif des Bauges a mobilisé une équipe de zootechniciens pour effectuer des observations du comportement alimentaire des génisses pendant plusieursétés sur l'alpage réhabilité,
Encadré 1. La question des liens entre pratiques pastorales et maintien de la biodiversité

Cette question se pose à l'échelle internationale : voir la critique des effets de l'élevage dans le rapport de la FAO, Livestock's Long Shadow: Environmental Issues and Options, 2006, pp. 180-218 (http://www.fao.org/ docrep $/ 010 / \mathrm{a} 0701 \mathrm{e} / \mathrm{a} 0701 \mathrm{e} 00 . \mathrm{htm}$ ) et la réponse de la League for Pastoral Peoples and Endogenous Livestock Development et de Drynet, intitulée Looking at the Bright Side of Livestock: Mobile Pastoralism and the Environment, 2007 (http://www.dry-net.org/uploaded_files/071201_The_Bright_Side_of_Life_final_english.pdf), dans laquelle ils présentent les pasteurs comme des " pilotes de la biodiversité ». En France, et plus particulièrement dans les Alpes, deux pôles se dégagent, séparés par toute une gamme de positions intermédiaires. D'un côté, certains considèrent le pastoralisme comme avant tout bénéfique à la préservation de la biodiversité (Béranger et Bonnemaire, 2008; Agreil et Greff, 2008), en raison de son rôle dans le maintien de milieux ouverts favorables aux espèces prairiales et à la diversité paysagère. De l'autre, le pastoralisme est au contraire tenu pour majoritairement néfaste, du fait des dégradations de la végétation (érosion des sols et surpâturage) et des conséquences négatives sur la faune sauvage (risques de transmission de pathologies, etc.) [Gauthier, 1992].

ainsi que des écologues pour y étudier les effets du pâturage sur la dynamique de végétation (Encadré 2). À chaque fois, il s'agit moins de produire un corpus systématique de savoirs proprement agrienvironnementaux (Micoud, 1993 et 2002), qui permettrait de fonder en toute connaissance de cause des pratiques pastorales respectueuses de la biodiversité, que de créer des dynamiques locales d'expérimentation et d'apprentissage collectif.

\section{Un succès mitigé}

Les acteurs entrepreneurs de conciliation ont donc mené un travail de conviction et d'enrôlement vis-à-vis tant de certains agents des parcs que des acteurs agricoles, enclins chacun à craindre toute forme de collaboration mutuelle. Par la suite, un certain nombre d'acteurs ont renoncé à un positionnement radical et se sont insérés dans des compromis : participation à la rédaction du SGEA et à sa mise en œuvre en Vanoise, participation aux rencontres du Mercantour, signature de contrats ou reprise d'alpages dans les Bauges. Néanmoins, ces déplacements dans l'espace frontière ont parfois été temporaires et certains sont restés sur des positions extrêmes, refusant d'y mettre les pieds.

Dans le cas de la Vanoise, la réalisation du SGEA a nécessité des rencontres entre le chargé de mission 


\section{Encadré 2. Les relations entre chercheurs et gestionnaires dans les Bauges}

Dans les Bauges, les relations entre gestionnaires d'espaces et chercheurs ont débuté avec le partenariat noué au milieu des années 1980 entre, d'une part, l'Office nationale de la chasse et de la faune sauvage (ONCFS) et l'Office national des forêts (ONF), gestionnaires de la réserve nationale de chasse et de faune sauvage située dans les hautes Bauges, et, d'autre part, le Laboratoire d'écologie alpine (LECA) du CNRS, dont les travaux portent sur les dynamiques de populations d'ongulés sauvages. Dans la réserve, créée dès 1913 afin de reconstituer les populations de chamois, les chasseurs ont joué - et jouent toujours - un rôle très important dans la collecte de matériaux divers utilisés par les chercheurs (crottes, cadavres, prélèvements et mesures effectués lors de captures), tandis que de nombreux stagiaires travaillent sur le site en période estivale. C'est ainsi qu'a été créé, à la fin des années 1990, le réseau Herbivorie, regroupant des chercheurs de diverses disciplines et institutions et les gestionnaires de la réserve autour d'un "site-atelier » partagé, l'alpage de l'Armène, situé dans la réserve et propriété de l'ONCFS : ce réseau facilite la réalisation des travaux de recherche grâce à l'existence d'infrastructures de logement et de manipulation et à l'accumulation de données. Si ce réseau s'est d'abord essentiellement intéressé aux interactions entre faune sauvage et milieux, certains gestionnaires ont conçu le projet de ramener des animaux domestiques sur l'alpage de l'Armène abandonné depuis 1962. Il entendaient notamment remédier à la diminution de la population de tétras-lyres en luttant contre l'embroussaillement et en restaurant un milieu propice à leur nidification. Rencontrant d'abord l'hostilité des éleveurs locaux, évincés de ce territoire à la fin des années 1950 (période à laquelle la réserve a acquis le statut de réserve nationale), ce projet a finalement bénéficié de l'investissement du parc naturel régional, créé en 1995, et de la mise en place de Natura 2000. Le retour d'un troupeau d'une cinquantaine de génisses depuis 2003, sous la conduite d'un éleveur local, a mené à la mobilisation d'une nouvelle équipe de chercheurs et de zootechniciens sur l'alpage de l'Armène (Meuret et al., 2006), ainsi qu'à la mise en place de suivis de l'évolution de la végétation et de la population de tétras-lyres sous l'effet de la présence de génisses. Pour un bilan des résultats intermédiaires obtenus, voir Jullien et al. (2007).

pastoralisme du parc et le salarié de la chambre d'agriculture impliqué dans l'instruction de ce dossier. Alors que beaucoup d'agriculteurs faisaient valoir initialement leur hostilité à l'interdiction du pâturage voulue par le parc sur certaines zones - refusant $\mathrm{qu}^{\prime}$ « on pousse les brebis pour laisser venir bouffer les bouquetins »-, des deux côtés, des acteurs ont accepté progressivement d'envisager des compromis. Le document lui-même, dont la page de couverture porte lenom et le logo des deux structures et où les enjeux agricoles, d'une part, et environnementaux, d'autre part, ont été remplacés par des enjeux " agrienvironnementaux », atteste de l'ouverture de cet espace de conciliation. Cette dynamique est cependant restée longtemps essentiellement institutionnelle et abstraite : le passage de la carte au terrain est renvoyé à un autre stade, à la suite de diagnostics pastoraux dont l'élaboration prendra du temps et apparaît, dans certains cas, incertaine et compliquée. Par ailleurs, le conseil scientifique du parc est resté marqué par des postures très hétérogènes concernant la possibilité de concilier activité agricole et maintien de la biodiversité. Un membre du conseil scientifique (également membre d'associations de protection de la nature ${ }^{6}$ ) a ponctuellement refusé d'assister à certaines séances du conseil, au motif qu'il y était trop question de pastoralisme et que, de plus en plus, la chambre d'agriculture dictait au parc ses positions. Il explique ainsi son désabusement vis-à-vis des politiques de rapprochement du monde agricole menées au sein du Parc national de la Vanoise (et d'autres espaces protégés), qu'il associe à une volonté stérile d'a paisement négligeant de plus les détériorations environnementales manifestes que dévoilent, selon lui, les travaux naturalistes. Ce type de discours, bien que partagé par certains agents de terrain, est disqualifié par d'autres membres du parc, qui estiment qu'il serait politiquement injustifiable (impossible à soutenir dans le contexte réglementaire et politique qui autorise, voire favorise, le pastoralisme) et dépassé sur le plan scientifique.

Dans le Mercantour, c'est un espace physique de rencontres qui s'est ouvert temporairement dans un endroit neutre (le lycée de Valdeblore) où personne n'était chez soi. Le nombre de participants aux rencontres (trois cents) a surpris les organisateurs eux-mêmes - alors que l'événement se déroulait dans un lieu particulièrement difficile d'accès. Les organisateurs avaient invité plusieurs représentants de l'agriculture à s'exprimer officiellement lors des rencontres et avaient multiplié les illustrations d'animaux domestiques sur la plaquette de la manifestation. En fin de compte, la participation de plusieurs représentants agricoles a été vécue par le parc comme un succès. Mais les agents de terrain du parc, sans avoir boycotté les rencontres, ne se sont quasiment pas exprimés, par peur, selon l'hypothèse de l'un d'eux, «de dire des choses qui fâchent». Certains participants ont ainsi estimé que le déroulement des rencontres avait été extrêmement cadré, voire " corseté », de manière à éviter tout conflit. Enfin, rien ne dit que l'espace frontière qui a été

\footnotetext{
6 Ligue pour la protection des oiseaux (LPO), Fédération Rhône-Alpes de protection de la nature (FRAPNA).
} 
constitué survive aux rencontres et se traduise en un ensemble de pratiques partagées.

Par rapport aux deux cas précédents, l'expérimentation entreprise dans le Parc naturel régional du Massif des Bauges se distingue par un degré plus grand de matérialisation. La constitution d'un espace frontière se concrétise par un alpage où se côtoient non seulement des éleveurs, des agents du parc et des chercheurs, mais aussi des animaux sauvages et domestiques, des plantes... Les acteurs doivent apprendre à faire avec des êtres dont ils n'étaient pas familiers. Par exemple, des éleveurs s'intéressent davantage aux "épines » que mangent leurs génisses. Des instruments, règles et équipements spécialement adaptés à cet espace et aux acteurs qui y évoluent sont mis au point, qui ne répondent pas nécessairement aux exigences développées dans les mondes d'origine (contrat spécifique de location de l'alpage, ajustement des dates de montée en alpage en fonction de l'état des ressources végétales, parcs pour les génisses...). L'adhésion à l'idée que la présence des troupeaux et le maintien de la biodiversité se confortent mutuellement a été particulièrement forte de la part de certains conseillers agricoles, qui ont joué un rôle important de relais de ce message en direction des éleveurs - même si tous ne sont pas convaincus. Des critiques ont aussi été exprimées par certains environnementalistes, membres de la FRAPNA, qui dénoncent un positionnement jugé trop agricole (conduisant au financement de travaux d'équipement agricole sur des crédits Natura 2000). Les avis concernant les bénéfices du pâturage sur le maintien des populations de tétras-lyres sont de même partagés.

\section{Les difficultés propres à la constitution des espaces frontières}

Globalement, les espaces frontières entre agriculture et protection de la nature sont donc loin d'avoir été marqués par des «migrations» massives d'acteurs qui viendraient s'y installer : il s'agit bien plutôt d'espaces précaires où circulent des acteurs de manière parfois temporaire. Comment expliquer cette fragilité spécifique des entreprises de conciliation?

\section{Usures et déceptions : des identités en tension}

Il n'est tout d'abord pas facile de s'investir dans les espaces frontières. Différents niveaux de tension caractérisent en premier lieu le vécu des acteurs entrepreneurs de conciliation. Ainsi, si certains d'entre eux expriment la satisfaction d'avoir vécu des changements importants ( "on a bougé »), d'autres témoignent plutôt de déceptions ou d'une forme d'usure face à la maigreur des changements constatés. Un premier niveau de tension est lié à l'ampleur des ambitions poursuivies et à la faiblesse des moyens (temps, financements) dont ils disposent au sein des structures qui les emploient. Les modifications successives des politiques agrienvironnementales et la précarité des possibilités de financement des contrats avec les agriculteurs ont, par exemple, été très largement soulignées comme génératrices de démotivation de la part des agriculteurs, mais aussi de leur propre part.

Un deuxième niveau de tension concerne les rapports des individus avec leurs mondes d'origine. Plusieurs cas de défection sont liés aux critiques, voire aux accusations de trahison, auxquelles les pionniers des espaces frontières se trouvent en butte. Un chargé de mission pastoralisme témoigne d'une "angoisse permanente " liée à la nécessité de s'assurer de la mobilisation durable des partenaires agricoles tout en subissant des oppositions du côté des environnementalistes. Un autre parle de « procès d'intention » de la part de certains agents de terrain qui accusent leurs collègues du siège d'être "propastoralistes ». Si ce type d'accusation n'a pas forcément amené les nouveaux habitants de l'espace frontière à se replier sur leur propre monde, ces problèmes génèrent une constante impression de ne pas tenir ses engagements d'un côté ou de l'autre.

Enfin, un dernier niveau de tension, rencontré par les entrepreneurs et par des acteurs ralliés à la conciliation, tient à la déception des espoirs qu'ils avaient nourris. En Vanoise, des acteurs se sont ainsi rapidement rapatriés dans leur monde d'origine, estimant que les déplacements qu'ils avaient consentis avaient été mal reconnus par l'autre camp. On notera tout particulièrement le retour de la part de l'entrepreneur principal du SGEA qui, après plusieurs années d'exercice, a préféré changer de fonction. La chargée de mission pastoralisme du Mercantour donne l'exemple de l'accumulation d'études sur les liens entre pratiques pastorales et biodiversité sans que celles-ci soient suivies d'effets, créant « une certaine lassitude très légitime au niveau des équipes de terrain ».

\section{Les difficultés de la production de connaissances entre recherche et gestion}

Un autre ordre de difficultés concerne ensuite les trajectoires des chercheurs mobilisés par les gestionnaires pour contribuer à la production de connaissances sur les liens entre agriculture et biodiversité. Pour les chercheurs concernés, le travail avec les gestionnaires d'espaces permet, certes, de disposer de bonnes conditions d'investigation en situation réelle et de s'investir dans des partenariats avec le monde de l'action en lien avec des «questions de société ». Cependant, circuler entre le monde de la gestion des espaces protégés et le monde académique ne va pas de soi. Ainsi, chercheurs et gestionnaires attendent des travaux menés ou exposés qu'ils produisent à la fois des connaissances valorisables sur un plan académique, des techniques utilisables pour la 
gestion et des éléments de savoir ou de pratique partageables avec les acteurs locaux. Un gestionnaire du Mercantour espère par exemple qu'un protocole mis en œuvre en collaboration avec des chercheurs apportera «un élément concret de dialogue avec l'éleveur sur quelque chose de tangible ", même si, " au niveau scientifique, ce n'est pas grand-chose ». Ce qui permet la publication dans les revues académiques ne coïncide pas nécessairement avec ce qui va emporter l'adhésion des acteurs locaux. Les types de preuves relayés par le chargé de mission du parc des Bauges (photos de génisses broutant des buissons prises par un des chercheurs impliqués, témoignage d'un agriculteur local sur la réhabilitation de l'alpage...) ne sont pas les éléments privilégiés par les publications des chercheurs concernés. On pressent donc les difficultés de la mise en phase des agendas de recherche entre les demandes des gestionnaires - qui impliquent de travailler longtemps dans le même territoire afin d'en connaître très bien les spécificités et l'histoire, de s'intéresser aux pratiques dans une approche systémique ou interdisciplinaire, de coconst-ruire les questionnements avec les acteurs locaux, etc. - et les exigences du monde académique - qui orientent plutôt vers une grande mobilité dans la carrière, une spécialisation toujours plus grande, ainsi que des pratiques expérimentales en conditions très contrôlées afin de satisfaire les standards de validité statistique imposés par nombre de revues scientifiques. Plusieurs chercheurs soulignent ainsi que ce niveau de contrôle est difficile voire impossible à obtenir lorsqu'on observe ou expérimente en milieu réel et que les variables de pâturage sont liées aux pratiques professionnelles d'un éleveur. La publication des résultats obtenus est alors particulièrement malaisée. Un chercheur témoigne du " grand écart » à réaliser sans cesse entre les exigences du monde de la gestion et celles de la recherche académique.

\section{Conclusion}

Ce travail permet finalement de contribuer à la compréhension sociologique des dynamiques de rapprochement entre monde agricole et monde de la protection de la nature ; il montre que celles-ci ne doivent pas être analysées seulement comme le résultat d'incitations institutionnelles, mais aussi comme des expérimentations sociotechniques mise en œuvre par des acteurs entrepreneurs d'une voie intermédiaire. Les chargés de mission pastoralisme des parcs étudiés ont en effet réussi à amener des acteurs (du côté des éleveurs comme du côté de leurs propres collègues) à abandonner des positions radicales pour envisager de nouvelles pratiques partagées de gestion des alpages. Leur action a reposé notamment sur la mobilisation d'acteurs du monde de la recherche, afin de produire ou d'exposer des connaissances susceptibles de fournir des repères partagés sur les relations entre conservation de la biodiversité et pratiques agricoles et d'amorcer un dialogue et des pratiques concertées.

Cependant, il ressort de notre travail que ces espaces frontières sont caractérisés par une fragilité, une précarité spécifique que nous avons cherché à comprendre. De part et d'autre de la frontière, beaucoup d'acteurs restent marqués par la crainte de «se faire avoir», de perdre leurs revendications et prérogatives propres. Des individus se sont par exemple très fortement investis pour faire exister la protection de la nature - ou pour défendre la profession agricole : tisser des liens avec ce qui a longtemps été considéré comme "l'ennemi » affaiblit ce à quoi ils se sont consacrés pendant des années ou des dizaines d'années. Les acteurs entrepreneurs eux-mêmes sont caractérisés par un vécu souvent difficile de leur « double casquette». De même, les chercheurs mobilisés par les gestionnaires connaissent des tensions et des risques de déception spécifiques. Afin d'être en mesure d'impulser des dynamiques d'apprentissage collectif, ils doivent affronter la difficulté que constitue la mise en phase des besoins des gestionnaires et des exigences académiques au sein de leurs agendas et de leurs carrières. Circuler dans les espaces frontières, qu'ils relèvent de la rencontre entre pastoralisme et protection de la nature ou de l'articulation entre recherche et gestion, demande ainsi beaucoup d'énergie et d'astuce sans que la reconnaissance soit toujours au rendez-vous.

Ce travail permet enfin de souligner, à côté de l'importance des dynamiques de production de connaissances (Granjou, 2011), celle aussi des dynamiques sociales, professionnelles et identitaires qui accompagnent l'investissement dans une voie médiane - entre agriculture et protection de la nature, comme entre recherche et gestion. Il importe en effet de saisir comment l'expérimentation technique (production de connaissances et de pratiques) s'ancre dans une expérimentation sociale (déploiement de trajectoires et d'identités professionnelles) [Meuret et Micoud, 2005]. Il importe enfin de saisir les tensions professionnelles et sociales que rencontrent les individus qui entreprennent de constituer et de faire fonctionner des espaces frontières, si l'on souhaite contribuer aux dynamiques de conciliation entre agriculture et protection de la nature.

\section{Références}

Agreil, A., Greff, N., 2008. Des troupeaux et des hommes en espaces naturels: une approche dynamique de la gestion pastorale, Vourles, Conservatoire Rhône-Alpes des espaces naturels.

Akrich, M., Callon, M., Latour, B. (Eds), 2006. Sociologie de la traduction: textes fondateurs, Paris, Presses des Mines.

Alphandéry, P., Billaud, J.-P., 1996. L'agriculture à l'article de l'environnement, Études rurales, 141-142, 9-19. 
Becker, H.S., 1985. Outsiders : étude de sociologie de la déviance, Paris, Métailié.

Béranger, C., Bonnemaire, J., 2008. Prairies, herbivores, territoires : quels enjeux?, Versailles, Quæ.

Blandin, P., 2009. De la protection de la nature au pilotage de la biodiversité, Versailles Quæ.

Galison, P., 1996. Computer simulations and the trading zone, in Galison, P., Strump, D.J. (Eds), The Disunity of Science: Boundaries, Contexts, and Power, Stanford, Stanford University Press, 118-157.

Gauthier, D., Gibert, P., Hars, J., 1992. Sanitary consequences of mountain cattle breeding on wild ungulates, in Spitz, F., Janeau, G., Gonzles, G., Aulagnier, S. (Eds), OngulésUngulates 91: Proceeding of the International Symposium, Toulouse, France, September 2-6, 1991, Paris, SFEPM / Toulouse, IRGM, 621-630.

Gieryn, T.F., 1983. Boundary-work and the demarcation of science from non-science: Strains and interests in professional ideologies of scientists, American Sociological Review, 48, 781-795.

Granjou, C., 2011. Integrating agriculture and biodiversity management: Between green legitimization and knowledge production, Sociologia Ruralis, 51, 3, 272-283.

Guston, D.H., 2001. Boundary organizations in environmental policy and science: An introduction, Science, Technology and Human Values, 26, 4, 399-408.

Jullien, J.-M., Agreil, C., Mestelan, P., Meuret, M., 2007. Les Bauges: à la reconquête des alpages, Faune sauvage, 278, 40-43 (http://www.oncfs.gouv.fr/IMG/file/habitats/milieux-montagnard/FS278_bauges.pdf).

Kohler, R.E., 2002. Landscapes and Labscapes: Exploring the LabField Border in Biology, Chicago and London, The University of Chicago Press.

Larrère, R., Lizet, B., Berlan-Darqué, M., 2009. Histoire des parcs nationaux: comment prendre soin de la nature?, Versailles, Quæ.

Lascoumes, P., Callon, M., Barthe, Y., 1997. Information, consultation, expérimentation: les activités et les formes d'organisation au sein des forums hybrides. Actes de la huitième séance du séminaire du programme Risques collectifs et situations de crise du CNRS, organisée par l'École nationale supérieure des mines de Paris le 12 juin 1997, Grenoble, MSH-Alpes.

Laslaz, L., 2006. Terre d'élevage ou «nature préservée» en zone centrale des parcs nationaux français des Alpes du Sud ?, Méditerranée, 107, 53-66 (mis en ligne le 2 décembre 2008 : http:/ / mediterranee.revues.org/index462.html ; consulté le 9 mai 2011).

Meuret, M., Micoud, A. (Eds), 2005. Quelles formes de connaissances socio-techniques dans les politiques de conservation de la nature impliquant l'élevage? Application aux mesures agrienvironnementales et à la procédure Natura 2000 en moyenne montagne. Rapport scientifique et technique, Programme de recherche pour et sur le développement régional : "Territoires, acteurs, agricultures en Rhône-Alpes », INRA/ CNRS.

Meuret, M., Débit, S., Agreil, C., Osty, P.-L., 2006. Éduquer ses veaux et génisses: un savoir empirique pertinent pour l'agroenvironnement en montagne?, Natures Sciences Sociétés, 14, 4, 343-352.

Micoud, A., 1993. Vers un nouvel animal sauvage : le sauvage «naturalisé vivant»?, Natures Sciences Sociétés, 1, 3, 202210.

Micoud, A., 2002. Des collections et des collectifs : les savoirs en jeu dans la gestion des sites Natura 2000. Communication au séminaire de la Délégation à l'agriculture, au développement et à la prospective (DADP), Montpellier, 17-18 décembre.

Pinton, F. (Ed.), 2006. La Construction du réseau Natura 2000 en France: une politique européenne de conservation de la biodiversité à l'épreuve du terrain, Paris, La Documentation française.

Selmi, A., 2006. Administrer la nature: le Parc national de la Vanoise, Paris, Éditions de la Maison des sciences de l'homme / Quæ.

Star, S.L., Griesemer, J.R., 1989. Institutional ecology "translations" and boundary objects: Amateurs and professionals in Berkeley's Museum of vertebrate zoology, 1907-39, Social Studies of Science, 19, 3, 387-420.

Reçu le 4 mai 2010. Accepté le 6 octobre 2011. 